\section{Immunité innée et reprogrammation, coup de main entre prix Nobel}

Laure Coulombel médecine/sciences, ADR Inserm Paris V,

2, rue d'Alésia, 75014 Paris, France.

laure.coulombel@inserm.fr
> La stratégie de reprogrammation d'une cellule différenciée en une cellule souche pluripotente (iPS), qui a valu le prix Nobel 2012 à Shinya Yamanaka, consiste à forcer dans cette cellule l'expression de facteurs de transcription caractéristiques de l'état pluripotent, dont OCT4, SOX2, KLF4 et cMYC (abrégé OSKM) [1]. Jusqu'à aujourd'hui, la technique la plus efficace consiste à transduire les cellules avec des vecteurs rétroviraux porteurs des séquences codant ces facteurs, ce qui expose la cellule hôte aux risques liés à une intégration virale en un endroit inadéquat. D'autres stratégies sont donc testées: addition quotidienne (environ 20 jours) d'ARN messagers (modifiés) codant ces facteurs, ou des protéines elles-mêmes, couplées à des peptides perméants (CPP pour cell permeant protein) pour franchir les membranes cytoplasmiques et nucléaires. Cette dernière stratégie est malheureusement très inefficace $(0,001 \%$ de cellules reprogrammées avec les CPPOSKM, versus 0,1 à $1 \%$ avec les vecteurs viraux) [2, 3]. Cette discordance dans l'efficacité de reprogrammation des protéines OSKM selon qu'elles sont délivrées via des vecteurs viraux ou sous forme CPP a intrigué l'équipe de John Cooke à Stanford; ce d'autant que les protéines CPP pénètrent dans pratiquement toutes les cellules humaines (> $80 \%$ ), sont transloquées dans le noyau où leur taux atteint en $24 \mathrm{~h}$ celui des protéines produites via I'intermédiaire de vecteurs viraux (ici pMX-Sox2 ou pMX-0ct4) [4]. Pourquoi y $a-t-i l$ reprogrammation dans un cas, et pas dans l'autre?
Si le taux des protéines OSKM exogènes atteint par les deux techniques est identique, en revanche l'activation transcriptionnelle de leurs gènes cibles (dont notamment les gènes pluripotents endogènes 0ct4, Sox2, Nanog) ne l'est pas. Dans les cellules transduites avec les seules protéines (CPP-SOX2 ou CPP-OCT4), elle est d'une part retardée (au-delà de $48 \mathrm{~h}$ ) et d'autre part beaucoup plus faible que dans les cellules transduites avec les vecteurs viraux $\mathrm{pMX}$-Sox2 ou pMX-0ct4, et ce quel que soit le jour de la mesure.

John Cooke fait alors une expérience clé : il ajoute aux CPP-SOX2 ou CPP-OCT4 un vecteur viral contrôle (contenant un ADNc non relevant, pMX-GFP). Le résultat est sans appel: l'expression des gènes pluripotents endogènes est restaurée au niveau qu'induit la transduction de pMX-Sox2 ou pMX-0ct4, et ce dès $24 \mathrm{~h}$. II y a donc un effet propre de la particule virale, indépendamment des ADNc qu'elle porte, et indépendamment de son intégration dans le génome de la cellule hôte, puisqu'un vecteur mutant incapable d'intégration a le même effet. En fait, le virus déclenche très rapidement après son entrée dans la cellule - avant sa rétrotranscription en ADN et son intégration dans le génome - l'intervention des protagonistes de l'immunité innée, et en particulier de TLR3 (toll like receptor), un récepteur de I'ARN double brin des virus. Les auteurs démontrent de façon convaincante que c'est l'activation de TLR3 qui, en synergie avec les facteurs OCT4 et SOX2, est responsable de la forte induction des gènes pluripotents endo- gènes (qui, ensuite, assurent la pérénnité de l'état pluripotent induit). Les TLR actionnent la première ligne de défense contre les pathogènes en reconnaissant des motifs protéiques associés aux pathogènes, mais aussi au LPS (lipopolysaccharide de la membrane des pathogènes) et aux acides nucléiques $[5,6]$. En aval des TLR, deux voies de signalisation sont classiquement activées: l'une, via MyD88 (myeloid differentiation primary response gene), est empruntée par tous les TLR sauf TLR3, qui, lui, emprunte une autre voie, via l'adaptateur TRIF, qui conduit, entre autres, à l'activation de NF- $\mathrm{KB}$.

Comme on pouvait s'y attendre, I'utilisation d'un agoniste non viral de TLR3 comme le poly I:C (polyinosinic polycytydylic acid) a le même effet que le virus lorsqu'il est utilisé avec les protéines pluripotentes (CPP-SOX2 ou CPP-OCT4). Les auteurs complètent leur étude en démontrant dans deux modèles cellulaires différents que l'activation de TLR3 (via le poly I:C) accélère et facilite (nombre de colonies iPS x 4) la reprogrammation de fibroblastes humains en iPS par les protéines CPP-OSKM ou les ARNm modifiés OSKM. La comparaison avec l'efficacité de reprogrammation via des vecteurs viraux n'est pas précisée.

La reprogrammation de cellules différenciées en IPS se fait via le remodelage de la chromatine. Est-ce à dire que TLR3 intervient dans ces modifications épigénétiques? C'est bien le cas. L'association de CPP-OCT4 et de poly I:C induit la triméthylation de la lysine 4 de l'histone 3 (H3K4me3), marque d'activation, 


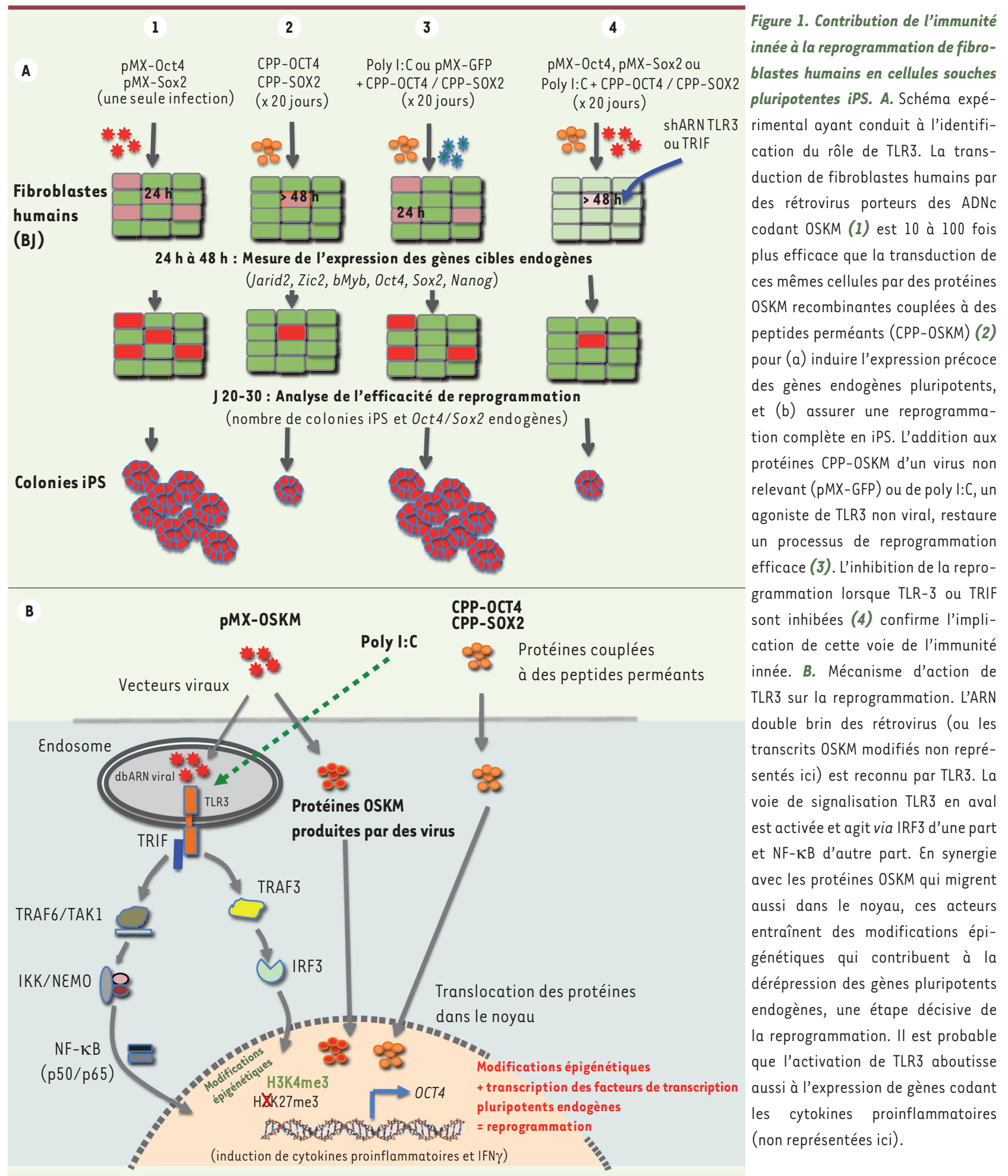

et élimine la triméthylation de la lysine 9 (marque répressive) de cette même histone (H3K9me3) au niveau des promoteurs des gènes pluripotents endogènes, dont Oct4, comme on l'observe dans les cellules transduites avec pMX-Sox2 ou
pMX-0ct4. En outre, indépendamment des CPP, poly I:C seul altère l'expression de nombreuses enzymes contrôlant le statut épigénétique, comme les histone déacétylases (HDAC) et plusieurs méthyltransférases, et agit sur la redis- tribution de $\mathrm{HDl}$, une hétérochromatine qui consolide la répression génique. Ces effets sont en partie sous le contrôle de la voie $N F-K B$, dont le gène est fortement induit en réponse à l'activation des TLR. 
En conclusion, les vecteurs viraux utilisés pour délivrer les facteurs pluripotents aux cellules différenciées sont plus qu'un véhicule passif. Ils contribuent, via leur activation de l'immunité innée, à l'efficacité de la reprogrammation en iPS, mais uniquement en synergie avec les facteurs de transcription de type OSKM. Curieusement, les auteurs n'évoquent que brièvement l'interféron de type 1 , qui est synthétisé lors de l'exposition à I'ARN viral, et donc lors de l'activation de TLR3, et n'en précisent pas le rôle. Au-delà du rôle facilitateur d'une voie de l'immunité innée - qui n'était pas anticipé - dans le processus de reprogrammation en iPS décrit ici, on peut s'interroger sur la signification de ces remaniements épigénétiques induits par l'activation de TLR3 (et probablement des autres $T L R$ ) dans d'autres circonstances. Les $T L R$, rappelons-le, sont des activa- teurs des voies pro-inflammatoires. Leur induction d'une certaine plasticité cellulaire (que l'éditorialiste de Science désigne sous le terme un peu inquiétant de ready-for-anything [7]) pourrait favoriser l'émergence d'autres altérations, moins «programmées », dont une transformation tumorale (on connaît les liens entre inflammation et cancer).

Comme nous vivons dans un monde pressé qui parle en langage Twitter and Co, les auteurs proposent de nommer ce processus «transflammation», ce qui évite de longues périphrases pour décrire la complexité de ce lien entre inflammation et reprogrammation. Qu'il plaise ou non, le terme risque d'être très utilisé s'il s'avère que cette découverte facilite et accélère la mise au point de techniques de reprogrammation efficaces et sûres. $\diamond$

Innate immunity and reprogramming, help between Nobel prize

\section{NOUVELle}

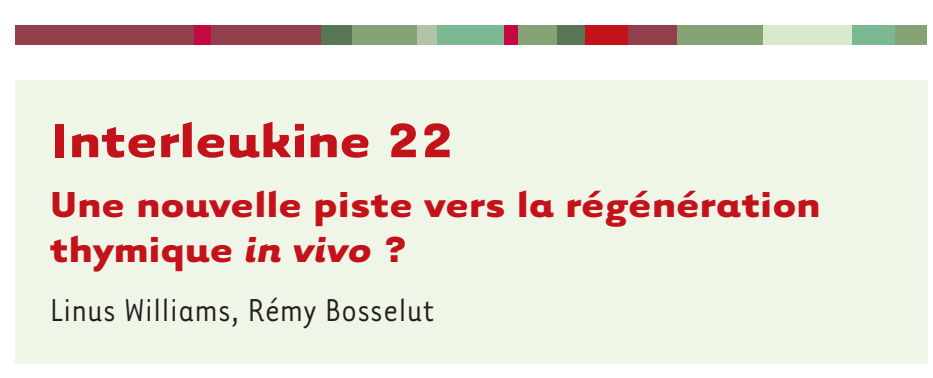

> Les lymphocytes $T$ sont un composant essentiel du système immunitaire, indispensable aux défenses contre les infections. La production de lymphocytes $T$ décroît rapidement après la puberté, contrairement à celle des autres leucocytes, ce qui implique que ces cellules ne sont pas remplacées si elles sont détruites chez l'adulte. Une étude récente ouvre toutefois de nouvelles perspectives dans ce domaine de la régénération thymique [1].
L'involution thymique des lymphocytes T chez l'adulte

La place centrale des cellules $T$ dans les réponses immunitaires provient non seulement de leurs effets directs (par exemple: lyse de cellules infectées par un virus), mais aussi de ce qu'elles coordonnent la fonction des autres cellules immunocompétentes (lymphocytes B, macrophages, etc.) [2]. Elles reconnaissent des antigènes spécifiques par l'intermédiaire d'un récepteur (TCR, T

\section{LIENS D'INTÉRÊT}

L'auteur déclare n'avoir aucun lien d'intérêt concernant les données publiées dans cet article.

\section{RÉFÉRENCES}

1. Coulombel L. Reprogrammation nucléaire d'une cellule différenciée : on efface tout et on recommence. Med Sci (Paris) 2007 ; 23 : 667-70.

2. $\mathrm{Kim} \mathrm{D}, \mathrm{Kim} \mathrm{CH}$, Moon JI. Generation of human induced pluripotent stem cells by direct delivery of reprogramming proteins. Cell Stem Cell $2009 ; 4: 472$ 6.

3. Zhou H, Wu S, Joo Jy, Zhu, et al. Generation of induced pluripotent stem cells using recombinant protein. Cell Stem Cell $2009 ; 4$ : 381-4.

4. Lee J, Sayed N, Hunter A, et al. Activation of innate immunity is required for efficient nuclear reprogramming. Cell $2012 ; 151: 547-58$.

5. Imler JL, Ferrandon D. Le printemps de l'immunité innée couronné à Stockholm. Prix Nobel de Médecine 2011 : Bruce A. Beutler, Jules A. Hoffmann et Ralph M. Steinman. Med Sci (Paris) $2011 ; 27$ : 1019-24.

6. Delneste $Y$, Beauvillain C, Jeannin P. Immunité naturelle: structure et fonction des Toll-like receptors. Med Sci (Paris) 2007 ; 23 : 67-74.

7. Vogel G. Stem cells. Immune reactions help reprogram cells. Science $2012 ; 338: 590$.

Laboratory of immune cell biology,

Center for cancer research,

National cancer institute,

National Institutes of Health,

37 Convent Drive, MD 20892-4 Bethesda,

Maryland, États-Unis.

remy@helix.nih.gov

cell receptor) dont les deux caractéristiques essentielles sont : une expression clonale (une cellule porte une seule spécificité antigénique) et une diversité qui résulte du réarrangement somatique, dans les précurseurs $T$, des gènes qui codent les chaînes de ce récepteur.

Comme toutes les cellules lymphoïdes, les lymphocytes $T$ dérivent de cellules souches hématopoïétiques localisées dans la moelle osseuse. Cependant, l'essentiel de leur développement prend 